3. Gosden RG, Richardson DW, Brown N, Davidson DW. Structure and gametogenic potential of seminiferous tubules in ageing mice. J Reprod Fertil 1982 ; $64: 127-33$.

4. Wang C, Hikim AS, Ferrini M, et al. Male reproductive ageing: using the brown Norway rat as a model for man Novartis Found Symp $2002 ; 242: 82-97$.

5. Peltola V, Huhtaniemi I, Metsa-Ketela T, Ahotupa M. Induction of lipid peroxidation during steroidogenesis in the rat testis. Endocrinology $1996 ; 137: 105-12$
6. Quinn PG, Payne AH. Steroid product-induced, oxygen-mediated damage of microsomal cytochrome P-450 enzymes in Leydig cell cultures. Relationship to desensitization. J Biol Chem 1985 ; 260 : 2092-9.

7. Chen H, Hardy MP, Huhtaniemi I, Zirkin BR. Age-related decreased Leydig cell testosterone production in the brown Norway rat. J Androl 1994 ; 15 : 551-7.

8. Chen H, Huhtaniemi I, Zirkin BR. Depletion and repopulation of Leydig cells in the testes of aging brown Norway rats. Endocrinology 1996 ; 137 : 3447-52.
9. Arimura A, Somogyvari-Vigh A, Miyata A, et al. Tissue distribution of PACAP as determined by RIA: highly abundant in the rat brain and testes. Endocrinology $1991 ; 129: 2787-9$.

10. Lacombe A, Lelievre V, Roselli C $\varepsilon$, et al. Delayed testicular aging in pituitary adenylate cyclaseactivating peptide (PACAP) null mice. Proc Natl Acad Sci USA 2006; 103 : 3793-8.

\title{
NOUVELLE
}

\section{Des senseurs pour contrôler le style de vie bactérien Le choix entre infection chronique ou aiguë}

Alain Filloux, Isabelle Ventre

\section{CNRS-IBSM-UPR9027,}

Laboratoire d'Ingénierie des Systèmes Macromoléculaires, 31, chemin Joseph Aiguier, 13402 Marseille Cedex 20, France.

\section{filloux@ibsm.cnrs-mrs.fr}

\section{le choix du style de vie bactérien}

Les bactéries sont largement répandues dans des niches environnementales ou infectieuses. Elles s'y développent essentiellement selon deux modes de vie que I'on appelle planctonique ou biofilm. Alors qu'en mode planctonique elles se déplacent librement sous forme de bactérie isolée, en mode biofilm elles se rassemblent pour former une communauté microbienne englobée dans une matrice d'exopolysaccharides et adhérente à une surface [1].

Parmi les raisons qui poussent les scientifiques à porter un intérêt croissant aux mécanismes moléculaires qui sous-tendent la formation de ces biofilms, il faut mentionner le rôle de ces populations bactériennes dans le développement des infections chroniques chez l'homme [2]. Pseudomonas aeruginosa est une bactérie pathogène très fréquemment associée aux infections nosocomiales. Chaque année en France, environ 4000 personnes dont le pronostic vital n'est pas menacé meurent d'une infection contractée à I'hôpital [3]. P. aeruginosa est impliquée dans nombre de ces infections, en particulier des pneumonies sévères. Dans ce contexte, la bactérie peut franchir la barrière du poumon, disséminer dans le flux sanguin et entraîner la mort du patient en quelques jours. Cette même bactérie est également l'agent responsable d'infections chroniques dans les poumons des patients souffrants de mucoviscidose. Ces patients sont tous colonisés dès l'âge de 3 ans par $P$. aeruginosa, et dans $80 \%$ des cas, la mort du patient est attribuée à une perte de la fonction pulmonaire due à l'infection chronique par $P$. aeruginosa des voies respiratoires. Dans ce cas, I'infection peut donc durer de nombreuses années et la bactérie n'atteint que très rarement le flux sanguin. Des évidences croissantes suggèrent que les infections chroniques soient associées au développement de biofilms très résistants au traitement antibiotique [2].

Si un même organisme est capable dans un contexte différent de prendre une décision vis-à-vis du style de vie qu'il doit adopter, c'est-a-dire croissance en biofilm versus croissance planctonique ou infection chronique versus infection aiguë, on doit de se demander ce qui dirige ce choix. Le processus pourrait être contrôlé par un réseau de régulation qui coordonne l'expression dans le temps et dans l'espace d'un ensemble de déterminants associés à la mobilité, l'adhérence ou la production d'exopolysaccharides, tous impliqués dans la formation du biofilm, ou bien, dans le cadre d'infections aiguës, de déterminants qui sont essentiels aux mécanismes associés à la cytotoxicité comme le système de sécrétion de type III (T3SS) [4].

Les bactéries possèdent des systèmes de détection qui permettent de percevoir les variations de conditions dans leur environnement afin de pouvoir en tenir compte pour changer l'expression de leur génome, et permettre ainsi l'induction ou la répression des gènes requis pour la meilleure adaptation au nouvel environnement détecté. Un système de régulation clé dans la mise en place de 
ce processus est le système de régulation à 2 composants [5]. Le senseur est une histidine kinase, qui possède un domaine de détection variable et spécifique pour un signal, et un domaine qui s'autophosphoryle suite à la détection du signal correspondant (Figure 1). L'information est ensuite transmise sur un régulateur de réponse par transfert du groupement phosphate depuis le senseur vers le domaine receveur du régulateur de réponse. La majorité des régulateurs

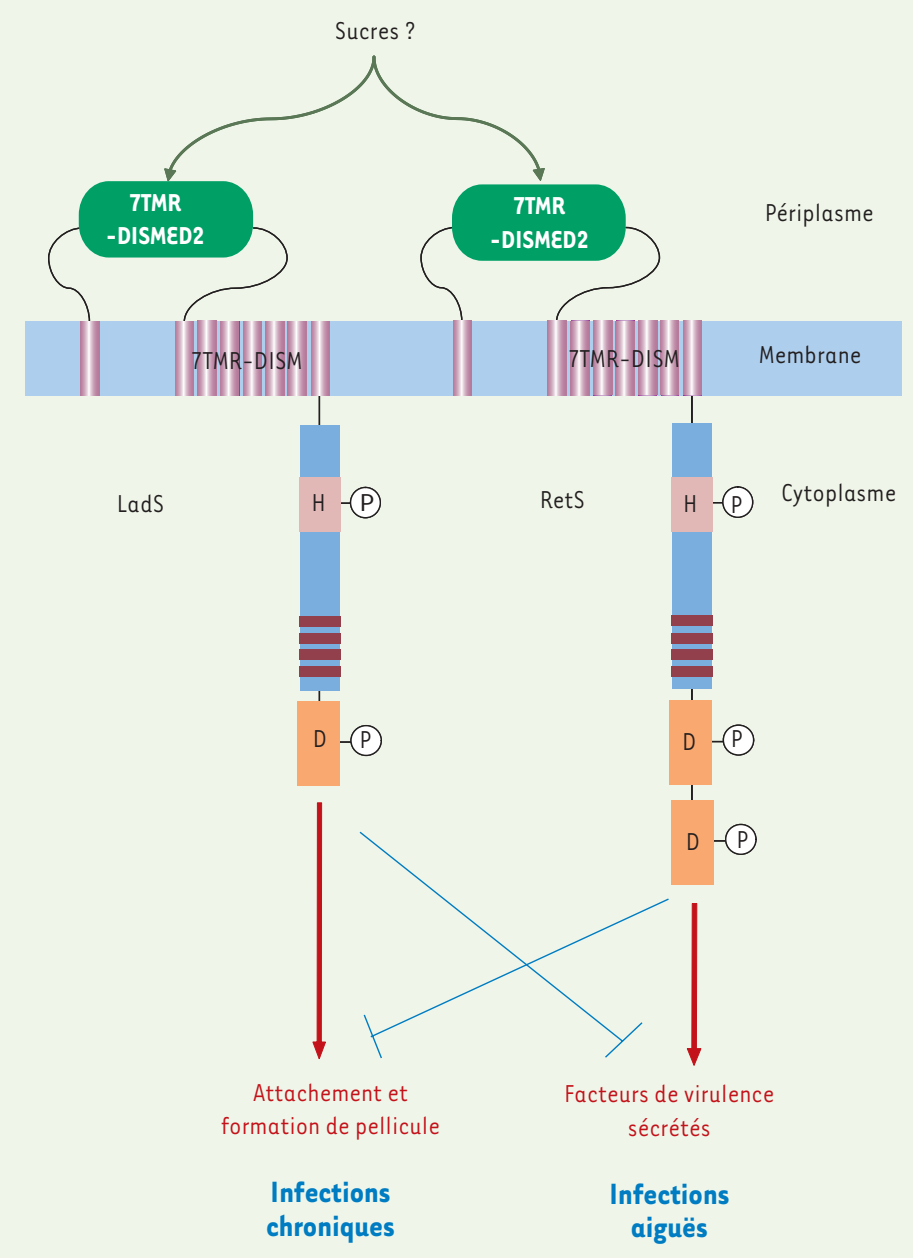

Figure 1. Les senseurs LadS et RetS contrôlent la transition entre biofilm et virulence. Les senseurs hybrides LadS et RetS sont insérés dans la membrane bactérienne par un domaine à 8 segments transmembranaires. Le $1^{\text {er }}$ segment est séparé du $2^{\mathrm{e}}$ par une boucle périplasmique, qui porte un domaine 7TMR-DISMED2, susceptible de détecter des sources carbonées. Le domaine transmetteur de la kinase s'autophosphoryle sur un résidu histidine $(H)$ après détection du signal. Le phosphate est transféré sur l'aspartate (D) du domaine receveur du senseur, puis est transmis vers le domaine receveur du régulateur de réponse qui ainsi activé va induire/réprimer un ensemble de gènes. $\mathrm{Si}$ les senseurs LadS et RetS sont structuralement similaires, RetS possède un domaine receveur supplémentaire. Le régulateur de réponse auquel aboutissent les deux senseurs pourrait être GacA et I'influence exercée sur les gènes cibles ferait intervenir le système RsmZ/RsmA (non représenté, voir texte). La cascade de régulation faisant intervenir LadS aboutit à une induction de gènes impliqués dans la formation du biofilm et l'attachement, alors qu'elle réprime les gènes du système de sécrétion de type III impliqué dans la cytotoxicité et la virulence. La cascade RetS contrôle ces mêmes gènes de façon strictement opposée. Cette balance génétique pourrait permettre de contrôler la transition entre phase d'infections chroniques ou aiguës. de réponse ainsi activés vont se lier à des régions d'ADN pour contrôler l'expression d'un panel de gènes appropriés.

Nos travaux ont récemment mis en évidence l'importance d'un senseur, LadS (lost adherence sensor) dans le contrôle de l'expression de gènes, comme les gènes pel, qui sont impliqués dans la production d'exopolysaccharides et le développement du biofilm $[6,7]$. Une souche de $P$. aeruginosa dont le gène ladS a été muté, se voit très sévèrement affectée dans sa capacité à former un biofilm (Figure 2A). De façon encore plus remarquable, ce même mutant ladS a un niveau de cytotoxicité augmenté (Figure $2 B$ ), corroboré par une forte augmentation de l'expression des gènes qui sont impliqués dans la mise en place de la machinerie de sécrétion de type III et ceux codant les toxines injectées dans la cellule hôte par le T3SS [6]. LadS peut donc être présenté ici comme un switch moléculaire qui dirige la transition entre biofilm et cytotoxicité, et donc infection chronique versus infection aiguë. De façon très remarquable, une étude publiée un peu plus tôt par le groupe du professeur Stephen Lory (Harvard Medical School, Boston, États-Unis) démontrait le rôle d'un autre senseur, RetS, dont l'influence sur les gènes pel et les gènes du T3SS est strictement opposée à celle de LadS [8]. Ainsi un mutant affecté dans le gène retS, voit sa capacité à former des biofilms augmentée (Figure 2A), à tel point que la souche est difficilement cultivable sous sa forme planctonique avec une forte tendance à s'agréger sur les parois du tube ou dans le milieu de culture. Cette tendance à former des biofilms est corroborée par la forte expression des gènes pel et psl, tous deux impliqués dans la production d'exopolysaccharides [8]. À l'inverse, la cytotoxicité du mutant retS est fortement diminuée (Figure 2B), comme l'indique également la faible expression des gènes du T3SS $[6,8]$.

Ces études nous révèlent donc l'existence de 2 senseurs qui gouvernent l'expression de gènes impliqués dans 
le biofilm et la cytotoxicité, mais qui le font d'une manière strictement opposée. On doit donc imaginer que si RetS contrôle l'expression de gènes en réponse à un signal présent dans les tissus de l'hôte lors d'une infection aiguë, maintenant ainsi l'activité du T3SS, la transition vers la formation du biofilm devrait faire suite à la disparition du signal perçu par RetS conjointement à l'apparition d'un autre signal perçu par LadS. La nature de ces signaux n'est pas encore connue, mais l'analyse des domaines détecteurs portés par ces 2 senseurs laisse penser qu'il pourrait s'agir de sucres [9].

Ce mécanisme de switch moléculaire et d'adaptation entre 2 modes de vie bactériens différents, gouverné ici par 2 senseurs indépendants, semble converger vers l'expression d'un gène codant pour un petit $A R N$ régulateur non codant appelé RsmZ [10]. En effet, l'expression du gène rsmZ est contrôlée de manière strictement opposée par LadS et RetS. Ainsi, la surproduction de RsmZ dans le mutant retS aboutit à la titration de la protéine régulatrice RsmA, qui a un impact important sur de nombreuses activités cellulaires liées à la virulence et la mobilité. Dans le mutant ladS, RsmZ est peu abondant laissant ainsi RsmA exercer son influence.

Le mécanisme de switch ainsi présenté (Figure 1) est certes simplifié, mais donne une bonne idée de l'intrication moléculaire des différents partenaires. II faut toutefois mentionner que d'autres systèmes à 2 composants devraient être associés à cette cascade de régulation. Le système GacA/GacS, par exemple, a été montré comme intervenant dans le contrôle de l'expression de rsmZ. De plus, le système RocARS, également connu comme SadARS [11], contrôle positivement la formation de biofilms, en particulier par l'expression de gènes codant des structures adhésives de type pilus (cup), mais dans le même temps contrôle négativement l'ex- pression des gènes du T3SS chez des bactéries qui se développent en mode biofilm [12,13].

\section{Conclusions et perspectives}

Nous faisons des choix chaque jour sur la base d'information dont nous disposons. Les bactéries font de même. Dans le contexte d'une infection, le pathogène doit prendre une décision lorsqu'il colonise son hôte. Doit-il se propager rapidement, provoquer une infection aiguë et éventuellement la mort de l'hôte, ou doitil persister au sein de I'hôte en s'établissant sous forme de biofilm et tenter d'échapper au système immunitaire. Ce petit jeu du DrJekyll and Mr Hyde, et la transition entre un état et l'autre, sont en grande partie dépendants des conditions environnementales. La disponibilité de certains sucres comme source de carbone, ou d'autres nutriments comme le fer, peuvent influencer la bactérie à persister sous forme de biofilms. La découverte de senseurs comme LadS et RetS, suggèrent qu'ils sont des éléments clés dans le processus de décision, et sont impliqués dans l'échantillonnage constant du milieu environnant. Les signaux que ces senseurs perçoivent sont encore mal connus, et nous devrons continuer à essayer de mieux comprendre tous les mécanismes moléculaires qui commandent le

B switch entre maladie aiguë ou chronique. Ces connaissances sont indispensables pour favoriser le développement de nouveaux anti-microbiens et améliorer le traitement des maladies infectieuses. $\diamond$

Two sensors to control bacterial life style: the choice between chronic or acute infection
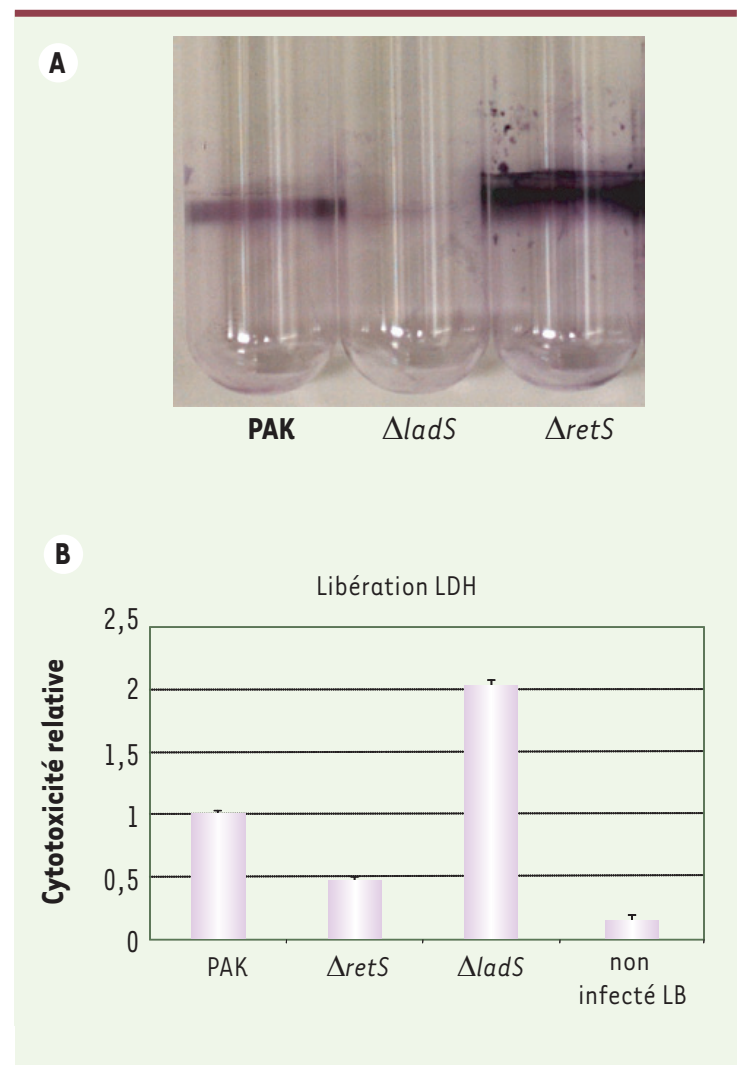

Figure 2. Formation du biofilm et cytotoxicité de Pseudomonas aeruginosa et des mutants isogéniques $\Delta l a d S$ et $\Delta$ retS. A. Les souches bactériennes sont cultivées à $30^{\circ} \mathrm{C}$ dans des tubes sans agitation en milieu minimum 63 additionné de casaminoacides. Après 8 heures de croissance, la culture est éliminée, le tube rincé et coloré avec du cristal violet. Les bactéries formant un biofilm sur les parois du tube peuvent ainsi être visualisées. On constate que le film formé par le mutant $\Delta r e t S$ est plus important que celui formé par la souche parentale (PAK) alors que le mutant $\Delta l a d S$ est incapable de s'accrocher à la paroi. B. La cytotoxicité relative des différents mutants par rapport à PAK est determinée par le \% de lactate déshydrogénase (LDH) libérée par des cellules $\mathrm{CHO}$ infectées par les différentes souches de $P$. aeruginosa. Le mutant $\Delta$ retS a un effet cytotoxique très faible puisque le niveau de LDH libérée est proche de celui observé avec des cellules non-infectées; en revanche, le mutant $\Delta$ ladS a un effet cytotoxique deux fois supérieur à PAK. 


\section{RÉFÉRENCES}

1. Hall-Stoodley L, Costerton JW, Stoodley P. Bacterial biofilms: from the natural environment to infectious diseases. Nat Rev Microbiol 2004 ; 2 : 95-108.

2. Costerton JW. Cystic fibrosis pathogenesis and the role of biofilms in persistent infection. Trends Microbiol $2001 ; 9: 50-2$

3. Coisne S. Les microbes se portent bien. La Recherche $2005 ; 389: 36-9$

4. Lee VT, Smith RS, Tummler B, Lory S. Activities of Pseudomonas aeruginosa effectors secreted by the type III secretion system in vitro and during infection. Infect Immun $2005 ; 73$ : 1695-705.

5. Rodrigue A, Quentin Y, Lazdunski A, et al. Twocomponent systems in Pseudomonas aeruginosa: why so many? Trends Microbiol 2000 ; 8 : 498-504.
6. Ventre I, Goodman AL, Vallet-Gely I, et al. Multiple sensors control reciprocal expression of Pseudomonas aeruginosa regulatory RNA and virulence genes. Proc Natl Acad Sci USA 2006 ; 103 : 171-6.

7. Vasseur P, Vallet-Gely I, Soscia C, et al. The pel genes of the Pseudomonas aeruginosa PAK strain are involved at early and late stages of biofilm formation. Microbiology 2005 ; 151 : 985-97.

8. Goodman AL, Kulasekara B, Rietsch A, et al. A signaling network reciprocally regulates genes associated with acute infection and chronic persistence in Pseudomonas aeruginosa. Dev Cell $2004 ; 7: 745-4$.

9. Anantharaman V, Aravind L. Application of comparative genomics in the identification and analysis of novel families of membrane-associated receptors in bacteria. BMC Genomics $2003 ; 4: 34$.
10. Heeb S, Blumer C, Haas D. Regulatory RNA as mediator in GacA/RsmA-dependent global control of exoproduct formation in Pseudomonas fluorescens CHA0. J Bacteriol 2002 ; 184 :1046-56.

11. Kuchma SL, Connolly JP, O’Toole GA. A threecomponent regulatory system regulates biofilm maturation and type III secretion in Pseudomonas aeruginosa.J Bacteriol 2005 ; 187 : 1441-54.

12. Kulasekara HD, Ventre I, Kulasekara BR, et al. A novel two-component system controls the expression of Pseudomonas aeruginosa fimbrial cup genes. Mol Microbiol $2005 ; 55$ : 368-80.

13. Vallet I, Olson JW, Lory S, et al. The chaperone/usher pathways of Pseudomonas aeruginosa: identification of fimbrial gene clusters (cup) and their involvement in biofilm formation. Proc Natl Acad Sci USA 2001 ; $98: 6911-6$.

\section{NOUVELle}

\section{La modélisation comme outil d'analyse pour la biologie du développement}

Christophe Godin, Jan Traas, Isabelle Bohn-Courseau, Pierre Barbier de Reuille

$>$ Du fait de leur nature sessile, les plantes doivent faire face aux variations importantes de leur environnement. Parmi les différentes stratégies qui permettent la survie dans un milieu souvent hostile, elles ont adopté un mode de développement très flexible. Cette plasticité du développement, spécifique du règne végétal, est permise principalement par une organogenèse continue. La plupart des plantes forment des embryons très rudimentaires, souvent constitués de quelques feuilles embryonnaires, une tige rudimentaire et une simple racine. Pourtant, à partir de ces embryons peuvent se développer des arbres de dizaines de mètres de haut, avec des architectures très complexes. Cette situation est donc très différente de celle des animaux supérieurs chez qui la majeure partie des organes est mise en place au cours de la vie embryonnaire.

\section{Des motifs phyllotaxiques remarquables}

L'organogenèse continue chez les plantes supérieures est assurée par des groupes de cellules non différenciées appelés méristèmes, mis en place durant l'embryogenèse. Au sein des méristèmes, quelques cellules se divisent régulièrement, produisant chacune une cellule identique à elle-même et une cellule destinée à se différencier: en cela, ces cellules sont équivalentes aux cellules souches animales. Les méristèmes sont donc des ensembles organisés de cellules indifférenciées qui, simultanément, s'auto-maintiennent et génèrent organes et tissus tout au long de la vie de la plante, parfois pendant des millénaires.

\section{Godin : Inria-Cirad}

Équipe Virtual Plants, Unité Mixte de Recherche Botanique et Bio-informatique de l'Architecture des Plantes, TA40/PSII, boulevard de la Lironde, 34398 Montpellier Cedex 5, France.

J. Traas: Inra, ENS-Lyon, Laboratoire de Reproduction et Développement des Plantes, 46, allée d'Italie, 69364 Lyon Cedex 7, France. I. Bohn-Courseau : Laboratoire de Biologie Cellulaire, Inra, Route de Saint-Cyr, 78026 Versailles Cedex, France. P. Barbier de Reuille : Inra, Équipe Virtual Plants, Unité Mixte de Recherche Botanique et Bio-informatique de l'Architecture des Plantes,

TA40/PSII, boulevard de la Lironde,

34398 Montpellier Cedex 5, France.

christophe.godin@sophia.inria.fr

jan.traas@ens-lyon.fr

Sans cesse en équilibre dynamique du fait des nombreuses divisions cellulaires, les méristèmes gardent cependant une structure stable au cours du temps et fonctionnent de façon très organisée [1]. L'exemple le plus frappant est sans doute la façon dont les organes, feuilles, 